\title{
Avaliação de Ambientes Educacionais: Análise do Estado da Arte em Sistemas Colaborativos e Tridimensionais
}

\author{
Adriana Doroteu Dantas ${ }^{1}$, Felipe Becker Nunes ${ }^{2}$, Thaís H. Chaves de Castro ${ }^{1}$, \\ Adanilton Rabelo de Andrade ${ }^{3}$, David W. Freitas Lima ${ }^{1,4}$ \\ ${ }^{1}$ Universidade Federal do Amazonas (UFAM) \\ ${ }^{2}$ Universidade Federal do Rio Grande do Sul (UFRGS) \\ ${ }^{3}$ Instituto Federal do Amazonas - Campus Eirunepé \\ ${ }^{4}$ Instituto Federal do Amazonas - Campus Manaus Zona Leste \\ \{dricadoroteu, nunesfb, thais.helena, adanilton.andrade, davidwfl\} \\ agmail.com
}

\begin{abstract}
Resumo. Este trabalho apresenta o estado da arte de duas vertentes de uma pesquisa que envolve a Avaliação de Ambientes Educacionais, no contexto Colaborativo e Tridimensional. Nesse trabalho foi conduzida uma Revisão Sistemática da Literatura, na qual foram utilizadas 6 bibliotecas digitais $e$ analisados 153 artigos. O objetivo dessa pesquisa é realizar uma análise entre as avaliações de interação existentes entre esses dois tipos de ambientes. Dessa forma, os resultados encontrados são relevantes para profissionais como programadores, designers e analistas, que podem repensar o planejamento dos projetos, antes e durante o desenvolvimento desses ambientes. Para os pesquisadores, a principal contribuição está nas discussões que podem nortear e embasar investigações futuras.
\end{abstract}

\begin{abstract}
This work presents the state of art of two aspects of a research that involves the Evaluation of Educational Environments in the Collaborative and Three Dimensional context. In this work was conducted a Systematic Review of Literature, in which, 6 digital libraries were used and 153 articles analyzed. The objective of this research is to perform an analysis between the interaction evaluations existing between these two types of environments. In this way, the results are relevant for professionals, such as programmers, designers and analysts, who can rethink project planning before and during the development of these environments. For the researchers, the main contribution is discussions that can guide and support future research.
\end{abstract}

\section{Introdução}

Os sistemas educacionais ou Sistemas de Gerenciamento de Aprendizagem (LMSs) são ferramentas de apoio à aprendizagem ou ensino, que se encontram cada vez mais presentes nas instituições. Sendo assim, os LMSs são responsáveis por organizar e disseminar o conhecimento entre a comunidade acadêmica. Tais sistemas educacionais podem ser somente um LMS para gerenciar conteúdos e interação ou possuírem 
mecanismos mais avançados de imersão e interação, como os ambientes $3 \mathrm{D}$, voltados para a aprendizagem de conteúdos específicos e jogos sérios.

Os mundos virtuais são definidos como ambientes online persistentes gerados por computador, onde as pessoas podem interagir, seja para o trabalho ou lazer, de forma comparável ao mundo real (Bainbridge, 2010). Na literatura, há diversos estudos sobre as avaliações de diferentes ambientes educacionais, como os trabalhos de Peixoto et al. (2015) e Rocha et al. (2015), em que, ambos autores trabalham avaliações em contextos educacionais diferentes. No entanto, quanto às possibilidades relacionadas às formas e modelos de avaliação da interação nesses ambientes, foi identificada uma maior escassez de trabalhos neste escopo.

A qualidade da interação proporcionada por esses ambientes é capaz de permitir ao aluno formas de comunicação suficientes para que o mesmo tenha um aprendizado satisfatório? A comunicação e interação de um ambiente educacional são características importantes a serem consideradas no momento da concepção dos sistemas, pois a falta dessas características pode prejudicar a aprendizagem dos alunos (Dantas et al. 2015).

Nesse contexto, a avaliação de ambientes educacionais tornou-se imprescindível para medir a qualidade de interação desses ambientes, em que esta revisão da literatura buscou verificar as avaliações em vários contextos na educação, tais como: avaliação na perspectiva do professor, na perspectiva do aluno e na perspectiva do tutor. De acordo com Behar (2010), avaliar é um ato que implica na definição de diversos critérios que devem ser estabelecidos pelo professor. Seja de forma presencial ou à distância, entende-se que a avaliação deve apoiar e incentivar o processo de construção do conhecimento, e não apenas servir como processo classificatório. Nesse contexto, Dantas et al. (2014) entende que é necessário fazer um planejamento do instrumento pedagógico, juntamente com o conhecimento e estratégias de desing, para atingir o sucesso efetivo da aprendizagem dos alunos por meio do ambiente educacional.

Outra forma de conseguir uma boa qualidade de interação desses ambientes é a utilização de ferramentas que propiciem a colaboração entre os alunos e professores, como LMS's configurados para privilegiar o uso de ferramentas de interação, como chats, fóruns e técnicas de debate. Isto é mostrado nos trabalhos de Kuntz et al. 2013 e Bassani et al. (2016). Nesse contexto, avaliação sistematizada das interações, considerando características colaborativas vêm se tornando cada vez mais necessários, no intuito de favorecer a aprendizagem colaborativa, que por sua vez também facilita a aprendizagem individualizada.

Este trabalho tem como objetivo caracterizar o contexto atual dos ambientes educacionais tridimensionais e colaborativos, a partir da condução de uma Revisão Sistemática de Literatura (RSL). Espera-se que os resultados obtidos possam apoiar profissionais do mercado de desenvolvimento, instituições de ensino e pesquisadores na construção de plataformas educacionais mais interativas. Nessa perspectiva, os artigos mais relevantes foram categorizados e analisados, indicando quais estratégias estão sendo utilizadas e quais os rumos para futuras pesquisas, no que se refere a avaliação de ambientes educacionais nos contextos de colaboração e tridimensionalidade. 


\section{Trabalhos Relacionados}

Durante esta pesquisa, diversos estudos foram avaliados, estando em algum nível relacionados ao tema central de avaliações de ambientes tridimensionais colaborativos no domínio educacional. Os métodos e técnicas empregadas pelos autores para o processo de avaliação é o foco dessa pesquisa. É importante descrever três obras que se situam no contexto apresentado nessa revisão sistemática.

O trabalho de Dantas et al. (2015) descreve um estudo de caso que aplica o método de avaliação de comunicabilidade em ambientes tridimensionais colaborativos no domínio educacional. Esse método deriva da Engenharia Semiótica e utiliza a ferramenta Sloodle. Os resultados deste trabalho descrevem quais elementos presentes na interface, que apresentam rupturas de comunicação durante a interação dos usuários.

No trabalho de Bowman et al. (2002) é realizado uma avaliação de usabilidade em um LMS tridimensional, apresentando comparações entre os métodos de avaliação e de acordo com três características principais: envolvimento de usuários representativos, contexto de avaliação e tipos de resultados produzidos. Já o trabalho de Fernandes et al. (2016), descreve um estudo sobre a avaliação de um ambiente tridimensional, que utiliza a heurística de Nielsen como forma de avaliar tal espaço.

No contexto de RS's encontrados e relevantes para esta pesquisa, o trabalho de Nunes et al. (2016) relata em sua RS sobre a aplicação de ambientes tridimensionais no contexto educacional, descreve diferentes tipos de abordagens educacionais e tecnológicas. No trabalho de Santos et al. (2012) descreve uma RS sobre as avaliações em ambientes colaborativos, relatando as especificidades e dificuldades encontradas para avaliar esses cenários e todos os métodos utilizados até a presente data para a avaliação de ambientes colaborativos.

O referido artigo descreve a RSL no contexto das avaliações de ambientes tridimensionais e colaborativos no domínio educacional utilizado atualmente pelos pesquisadores. Os principais resultados descritos são quanto às diferentes formas de avaliar esses ambientes, o número de trabalhos que utilizam o ambiente tridimensional para interagir colaborando com outros usuários, sendo as discursões pertinentes aos contextos estudados na RSL, além de contextualizar a avaliação da colaboração desses cenários tão específicos para a educação.

\section{Procedimentos Metodológicos}

A metodologia utilizada para a condução da Revisão Sistemática está embasada nas concepções de Kitchenham e Charters (2007), Biolchini et al. (2005) e Basili et al, (1988). Para Kitchenham e Charters (2007), este processo consiste em identificar, avaliar e interpretar todas as pesquisas disponíveis relevantes para uma questão específica, área temática ou fenômeno de interesse. A Revisão da Literatura é uma pesquisa explícita e rigorosa, que procura identificar o conhecimento científico em uma determinada área, por meio da coleta, combinação e avaliação crítica de descobertas de diversas abordagens já realizadas (Biolchini et al. 2005). Para ambos autores, as fases necessárias para a elaboração da revisão são 3: Planejamento da Revisão; Procedimentos de Condução, Extração e Exclusão; Procedimentos de elaboração de relatórios. 
VI Congresso Brasileiro de Informática na Educação (CBIE 2017)

Anais do XXVIII Simpósio Brasileiro de Informática na Educação (SBIE 2017)

\subsection{Protocolo}

$\mathrm{Na}$ fase de planejamento, a definição do protocolo é a primeira tarefa a ser elaborada. Assim como a questão de pesquisa a ser investigada com a descrição do objetivo. $O$ protocolo da pesquisa segue o paradigma conforme Goal-Question-Metric (GQM) (Basili et al, 1988).

\section{Tabela 1 - Protocolo da Pesquisa}

\begin{tabular}{|ll|}
\hline Analisar & Publicações científicas, periódicos e revistas \\
\hline Com o propósito de & Caracterizar \\
\hline Em relação a & $\begin{array}{l}\text { Identificação de técnicas e métodos que avaliam a interação em } \\
\text { ambientes 3D colaborativos no domínio educacional. }\end{array}$ \\
\hline Do ponto de vista dos & Dos pesquisadores \\
\hline No Contexto & Acadêmico \\
\hline
\end{tabular}

Fonte: elaborado pelos autores deste trabalho

No protocolo de pesquisa descrito na Tabela 1, deve estar presente o que se quer coletar, o problema da pesquisa e a resposta da mesma deve ser central, pois a partir dessa questão será delimitada a busca de forma precisa dentro do domínio de interesse. A string de busca formada por uma combinação de palavras-chave relacionadas a questão de interesse é elaborada nessa fase. Após os resultados encontrados são delimitados os critérios de extração e de exclusão dos artigos vistos.

\subsection{Questão de Pesquisa}

O objetivo é identificar um conjunto de técnicas e/ou métodos de avaliação de interação em ambientes 3D. Sendo as questões de pesquisa investigadas nesta revisão:

- QP1 - Quais os métodos e/ou técnicas de avaliação da interação em ambientes $3 \mathrm{D}$ colaborativos têm sido empregadas pelos pesquisadores?

- QP2 - Quais são as dimensões avaliadas quanto a interação colaborativa?

\subsection{Definição da Busca}

A pesquisa será a partir de uma biblioteca digital por meio de buscas avançadas, o engenho de busca presente na maioria das fontes de pesquisa. A busca manual ocorreu no Google acadêmico e na Revista de Informática na Educação. Na tabela 2 serão mostradas as fontes consideradas no escopo desta pesquisa.

Tabela 2 - Lista das Bibliotecas Pesquisadas

\begin{tabular}{|l|l|l|}
\hline \multicolumn{1}{|c|}{ Nome da Fonte } & \multicolumn{1}{c|}{ Link } & Tipo de Pesquisa \\
\hline Biblioteca ACM & http://dl.acm.org/ & Máquina de Busca \\
\hline Biblioteca IEEEXplore & https://www.ieee.org & Máquina de Busca \\
\hline Elsevier Science Direct & http://www.sciencedirect.com/ & Máquina de Busca \\
\hline Scopus & https://www.scopus.com & Máquina de Busca \\
\hline Google Acadêmico & http://scholar.google.com.br/ & Máquina de Busca \\
\hline $\begin{array}{l}\text { Revista Informática na Educação, } \\
\text { SBIE, WIE e Wavalia }\end{array}$ & http://www.brie.org/pub/index.php/rbie & Máquina de Busca \\
\hline
\end{tabular}

Fonte: elaborado pelos autores deste trabalho

Os termos utilizados nesta revisão sistemática foram agrupados em três grupos, que combinados entre si, formam a string de busca. Seguindo os critérios de 
Kitchenham (2004), a inserção de termos é importante para coletar resultados relevantes, para tanto foi dividido em 3 termos: população, intervenção e resultados (PICOC). Os termos estão escritos em inglês, português e espanhol, por ser o idioma utilizado pelas máquinas de busca consideradas no escopo deste trabalho. Foram geradas para a pesquisa três strings de busca com os mesmos termos, mas com idiomas diferentes (inglês, espanhol e português) para coletar um maior número de artigos:

("educational OR education OR serious OR educação OU ensino OU aprendizagem OU educação OU ensino OU aprendendo) AND (method OR technique OR interaction OR evaluation OR assesment OR colaboration) AND ("mundos virtuais" OU "ambientes virtuais" virtuais 3D "OU "ambientes virtuais multi-usuários" OU ambientes virtuais colaborativos" OU" mundos virtuais" "OU" ambientes imersivos "OU metaverses OU" mundos virtuais digitais 3D").

\subsection{Seleção dos Estudos}

A Tabela 3 apresenta a descrição dos primeiros resultados da busca realizada nas seis bibliotecas o resultado total dos artigos foram de 458. Tendo como base o guia de Kitchenham e Charters (2007), foram executados alguns passos para obter os estudos primários.

1. Análise de títulos: para a eliminação dos estudos com duplicidade e que eram irrelevantes, foi analisado somente os títulos dos 458 artigos encontrados. Após essa etapa foram eliminados 227 artigos, restando 231 artigos.

2. Análise do resumo: nos estudos que não estavam relacionados com as questões de pesquisa, foram analisados os seus resumos, resultando em um total de 231 artigos. Após essa etapa, foram eliminados outros 78 artigos, restando 153 artigos.

3. Análise rápida do texto: para os estudos que atendiam aos critérios de inclusão, uma leitura rápida (leitura em z) dos 153 artigos foi realizada. Após essa etapa, foi detectado que todos os artigos descreviam uma avaliação em ambientes educacionais, no entanto, poucos artigos respondiam à questão de pesquisa. Foram excluídos 117 artigos, ficando apenas 36 artigos.

4. Leitura detalhada: foram analisados detalhadamente os 36 artigos restantes e excluídos os trabalhos que se enquadravam nos critérios de exclusão. Desta forma, foram excluídos mais 19 artigos, restando apenas 17 artigos.

Para que fossem encontrados trabalhos relacionados às questões de pesquisa foi descrito o critério de inclusão (CI) em 4 itens, focando sempre a área de interesse da avaliação e os critérios de exclusão (CE) foram relacionados em apenas 2 itens. Esses critérios estão descritos abaixo:

- CI1. Podem ser selecionadas publicações que apresentam métodos e técnicas de avaliação de interação em ambientes em 3D colaborativos.

- CI2. Podem ser selecionadas publicações que descrevam uma avaliação de interação de ambientes em 3D colaborativos. 
VI Congresso Brasileiro de Informática na Educação (CBIE 2017)

Anais do XXVIII Simpósio Brasileiro de Informática na Educação (SBIE 2017)

- CI3. Podem ser selecionadas publicações que apresentam uma abordagem usada em uma organização desenvolvedora de ambientes em 3D para avaliar a interação dessas aplicações.

- CI4. Podem ser selecionadas publicações que apresentam métodos e/ou técnicas de interação em ambientes 3D.

- CE1. Não serão selecionadas publicações em que não atendem aos critérios de inclusão.

- CE2. Não serão selecionadas publicações que não tenham disponibilidade de conteúdo para leitura e análise dos dados (especialmente em casos, onde os estudos são pagos ou não disponibilizados pelas máquinas de buscas).

\section{Discussão dos Resultados}

O resultado no contexto de tipos de avaliação em ambientes educacionais foi bastante amplo, dando o retorno de 153 artigos. A quantidade de artigos abrange todos os ambientes educacionais, dentro e fora da questão de pesquisa, lembrando que a questão de pesquisa se concentra em avaliações de ambientes tridimensionais colaborativos.

As avaliações descritas nos artigos em sua maioria, $25 \%$ de $100 \%$ são em relação a aprendizagem de conteúdo e aprendizagem do sistema. A avaliação de usabilidade mostra $22 \%$ do total. Outros tipos de avaliações estão divididos entre vários métodos de avaliações em IHC e outros tipos de avaliações, o resultado dessa fatia no gráfico retornaram $20 \%$ do total, e apresentam formas diferentes de avaliar um ambiente, por exemplo, no contexto de tutores, como mostra o trabalho de Lima et al. (2014), que aborda a perspectiva de avaliação processual envolvendo tanto os tutores quanto os instrumentos e métodos adotados no desenvolvimento das atividades tutoriais.

Dentro do cenário de avaliações descritos acima as avaliações em ambientes tridimensionais colaborativos que se concentra na questão de pesquisa principal foi respondida com o retorno de 36 artigos dos quais 17 focam e tratam da avaliação em ambientes com cenários colaborativos e tridimensionais no domínio educacional.

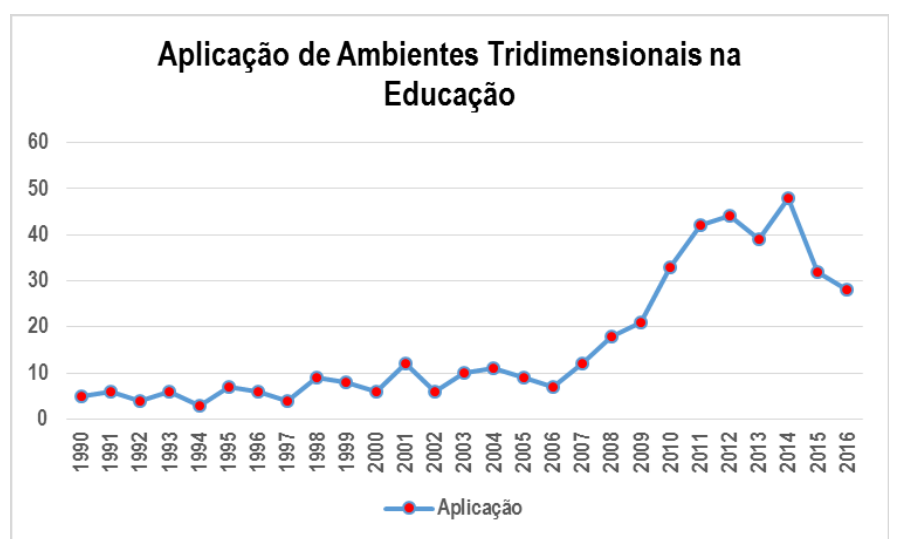

Figura 1 - Gráfico das Aplicações de Ambientes Tridimensionais na Educação

\section{Fonte: elaborado pelos autores}

A Figura 1 mostra que a incidência da aplicação de ambientes tridimensionais no contexto educacional vem crescendo nos últimos anos. Esse quadro só reforça a 
VI Congresso Brasileiro de Informática na Educação (CBIE 2017)

Anais do XXVIII Simpósio Brasileiro de Informática na Educação (SBIE 2017)

afirmação de autores como Girotto (2016) e Blanco et al. (2016), que falam das principais formas de interação mais atuais são os ambientes tridimensionais, juntamente com os ambientes de realidade virtual aumentada, esses ambientes estão sendo cada vez mais aplicados na educação com o intuito de chamar a atenção do aluno e facilitar a sua aprendizagem durante a experiência real de cenários imersivos (Anjos, 2016).

\subsection{Extração dos Dados}

A primeira questão de pesquisa que havia sido definida foi:

- QP1 - Quais os métodos e/ou técnicas de avaliação da interação em ambientes 3D têm sido empregadas pelos pesquisadores?

Os artigos que respondem as questões de pesquisa e que foram extraídas no final da revisão sistemática, apresentam os tipos de métodos utilizados como forma de avaliar os ambientes tridimensionais colaborativos no domínio educacional. O gráfico da Figura 2, abaixo apresenta a resposta da questão de pesquisa e apresenta um total de 28 trabalhos sobre os tipos de avaliação para ambientes tridimensionais.

É possível notar que o teste de usabilidade foi o tipo de avaliação mais utilizada para avaliar ambientes tridimensionais, por ser um termo mais comum no cenário das avaliações, o contexto de uso vem se tornando cada vez mais importante. De acordo (Barbosa e Silva, 2010) a eficácia diz respeito aos usuários alcançarem seus os objetivos corretamente através da interação com o sistema e a eficiência refere-se aos recursos necessários para os usuários atingirem seus objetivos.

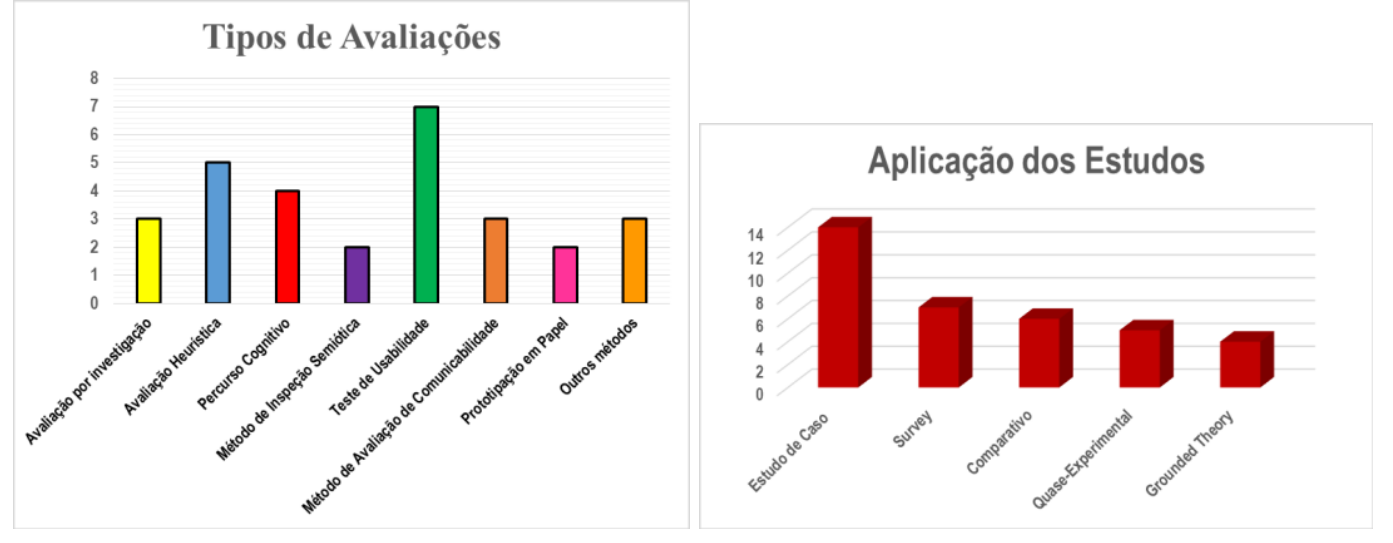

Figura 3 - Gráfico dos Tipos de Avaliações em IHC no Cenário Educacional Fonte: elaborado pelos autores

Os métodos e técnicas utilizadas para avaliar a interação de ambientes tridimensionais colaborativos foram divididos em 2 (dois), um baseado nas técnicas e métodos da área de IHC e o outro em técnicas específicas de ambientes tridimensionais, um exemplo disso é o trabalho de Bowman et al. (2004) que relata sobre as especificações de avaliação da tridimensionalidade tais como: navegação, manipulação e controle do sistema. Ainda no cenário de estudos encontrados na revisão sistemática foram classificados em 7 tipos de avaliação e 5 tipos de aplicação em estudos.

Ainda a Figura 2 apresenta que o estudo de caso foi o tipo de avaliação que ocorreu em maior incidência dentre os tipos de estudos conduzidos, os trabalhos de 
Dantas et al. (2014) e de Nunes et al. (2016) abordam justamente experiências com estudos de caso exploratórios sobre a avaliação de cenários tridimensionais colaborativos no domínio educacional. O gráfico abaixo mostra que o survey é o segundo método mais utilizado sendo $28 \%$ do total de trabalhos, seguido de estudos comparativos entre os métodos de avaliação.

- QP2 - Quais são as dimensões avaliadas quanto a interação?

Para a resposta de tal questão de pesquisa foram catalogados os trabalhos em 3 (três) grupos específicos: A colaboração, comunicação e a socialização, conforme visto na Figura 3. Retornaram 28 artigos que contemplam a resposta da questão de pesquisa 2. A colaboração entre os usuários é item imprescindível a ser avaliado em um ambiente educacional, pois favorece a elaboração de atividades em grupo e a aprendizagem colaborativa. Os tipos de atividades disponíveis nos ambientes precisam proporcionar a colaboração entre todos os atores presentes no mesmo espaço (Buchinger et al. 2013). O tema é tão atual e relevante que sua importância é discutida nos trabalhos de Fuks et al. (2012), Cook et al. (2008), Schauer e Zeiller (2011).

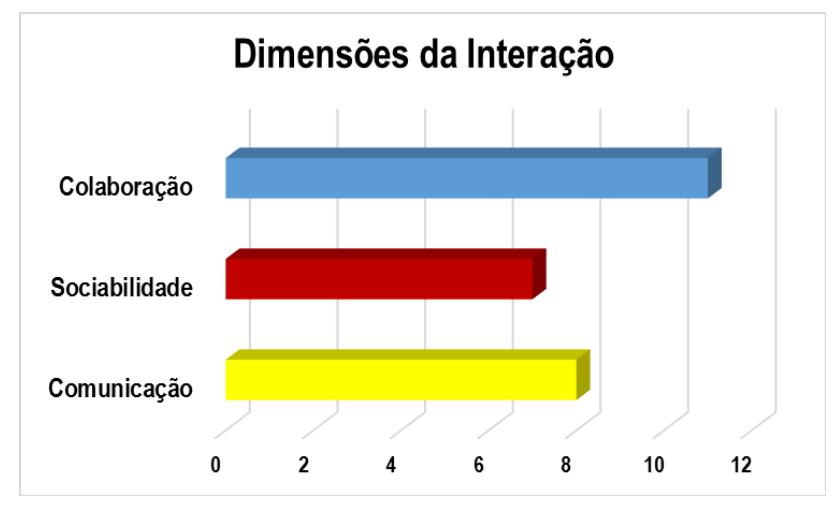

Figura 3 - Gráfico Resposta da Questão de Pesquisa 2

Fonte: elaborado pelos autores

A comunicação é um dos itens mais vivenciados durante a interação de ambientes tridimensionais. Conceitos e ideias inexistentes sobre objetos e funções trazem a necessidade de uma interpretação rápida, que deve ser feita sobre os artefatos presentes no ambiente para concluir os objetivos propostos (Dantas et al. 2015). Nesse contexto, o trabalho de Moreira et al. (2016) relata sobre a temática da colaboração em ambientes educacionais e da sua contribuição para a aprendizagem nos dias de hoje.

A sociabilidade em ambientes de aprendizagem é uma característica que dá ao usuário o poder de se comunicar e se relacionar com os demais participantes de um mesmo sistema ou jogo. O trabalho de Chagas et al. (2011) aborda sobre as várias temáticas da sociabilidade com um jogo tridimensional educacional colaborativo.

\section{Considerações Finais}

O resultado da revisão sistemática mostrou um grande número de avaliações voltadas a aprendizagem, e um baixo número de publicações preocupadas com as avaliações de interações em ambientes tridimensionais colaborativos e alguns trabalhos voltados aos projetos desses ambientes. No entanto, a preocupação com a falta dos aspectos de comunicação, socialização e um design que facilite a interação em ambientes educativos 
VI Congresso Brasileiro de Informática na Educação (CBIE 2017)

Anais do XXVIII Simpósio Brasileiro de Informática na Educação (SBIE 2017)

podem prejudicar a aprendizagem dos alunos, tornando essa uma discussão importante para a comunidade acadêmica.

Os resultados da quantidade de trabalhos que abordam o crescente número de uso de ambientes tridimensionais na educação mostram que a interação desses ambientes é importante como forma de propiciar a aprendizagem dos alunos. A produção e o desenvolvimento de ambientes cada vez mais interativos ressaltam a necessidade de entender, compreender e estudar esses cenários tão peculiares.

Em se tratando de ambientes cujo principal objetivo é a aprendizagem do aluno, a finalidade da avaliação deve propor formas de facilitar a absorção de conhecimento, trabalhando nos problemas encontrados após as análises de quem realmente os utiliza que são os alunos. Seria importante descrever também até que ponto a ruptura de comunicação de um ambiente educacional prejudica a aprendizagem dos alunos, o que seria uma contribuição relevante em uma pesquisa futura.

Os termos estudados na segunda questão de pesquisa são formas e técnicas inseridas no ambiente que podem proporcionar a aprendizagem, como a colaboração, socialização e comunicação. Todas as técnicas mencionadas precisam constar nos cenários desses ambientes, bem como disponibilizar atividades que possam conduzir o aluno a interação. Nesse sentido, o resultado da RSL aborda a importância do professor na condução da criação das atividades e disposições de conteúdos, além disso, a equipe pedagógica precisa contribuir com a elaboração desses cenários de interação.

O resultado da revisão para os desenvolvedores traz à tona a responsabilidade do desing de interação no desenvolvimento de ambientes educacionais e de como esses profissionais precisam focar no aperfeiçoamento das ferramentas de colaboração existentes e trabalhar na elaboração de novos instrumentos que possam conduzir a participação efetiva do aluno nesses cenários.

\section{Referências}

Anjos, A. M.; Fátima, L. S. N.; Tori, R. Avaliação de habilidades sensório-motoras em Ambientes de Realidade Virtual para treinamento médico: uma Revisão Sistemática. Simpósio Brasileiro de Informática na Educação 2016.

Bainbridge, W. S. (2010). Online Worlds: Convergence of the Real and the Virtual. In Human-Computer Interaction Series, Springer-Verlag, London Limited.

Barbosa, S.D.J., Silva, B.S. Interação Humano-Computador. (2010). Editora Campus. Rio de Janeiro: Elsevier, 2010. (Série SBC, Sociedade Brasileira de Computação). ISBN 978-85-352-3418-3

Basili, V.R. and Rombach, H.D.: The TAME project: Towards improvement-oriented software environments, in IEEE transactions on Software Engineering pp. 758-773, 1998.

Bassani, P. B. S., dos Reis, A.N., Dalanhol, D. (2016). Análise da colaboração em ambientes digitais para compartilhamento de atividades de aprendizagem: Uma perspectiva com base em Learning Design.In: V Congresso Brasileiro de Informática na Educação (CBIE). Anais do XXVII Simpósio Brasileiro de Informática na Educação (SBIE). Uberlândia-MG, Brasil. 
VI Congresso Brasileiro de Informática na Educação (CBIE 2017)

Anais do XXVIII Simpósio Brasileiro de Informática na Educação (SBIE 2017)

Behar, P.B., Longaray, A. N. C., Bernardi, M., Ribeiro, A.M.R., da Silva, K. K. A. (2010). AVALEAD: A construção de um Objeto de Aprendizagem sobre Avaliação em Educação a Distância. In: Centro Interdisciplinar de Novas Tecnologias na Educação - CINTED-UFRGS. Porto Alegre - RS, Brasil.

Biolchini, J.; Mian, P. G.; Natali, A. C. C.; Travassos, G. H. (2005) Sytematic review in software engineering. Relatório Técnico, RT-ES 679/05 System Engineering and Computer Science Dept., COOPE/UFRJ.

Blanco, M.M., Bjørn, P., De Angeli, A. (2016). Fostering Cooperative Activism through Critical Design. In: Computer Supported Cooperative Work - CSCW '16, Portland OR, USA.

Bowman, D.; Addison, W. - 3D user interfaces: theory and practice. Boston:, 2004.

Bowman, D.A., Gabbard, J. L., Hix, D. (2002). A Survey of Usability Evaluation in Virtual Environments: Classification and Comparison of Methods, Presence: Teleoperators and Virtual Environments, August 2002, Vol. 11, No. 4, Pages 404424 doi:10.1162/105474602760204309. Bowman,D; Kruijff,E; JJ LaViola Jr, IP Poupyrev - Virtual reality: how much immersion is enough. New York, 2004.

Buchinger, D., Hounsell, M. DA S., 2013. Jogos Sérios Competitivos-Colaborativos: Uma Mapeamento Sistemático da Literatura. In: Proceedings of the XXIV Simpósio Brasileiro de Informática na Educação - SBIE, 2013 Campinas, 275-284.

Chagas, Daniel A., Rafaela P. Lisboa, Furtado, Elizabeth S. - Framework MAAVA Metodologia de Avaliação de Ambientes Virtuais de Aprendizagem. XXV Simpósio Brasileiro de Informática na Educação - SBIE, 2011, 275-284.

Cook, N. (2008). "Enterprise 2.0 - How Social Software Will Change the Future of Work". Aldershot: Gower.

Da Rocha, R.V., Bittencourt,I.I., Isotani, S. (2015). Análise, Projeto, Desenvolvimento e Avaliação de Jogos Sérios e Afins: uma revisão de desafios e oportunidades. In: Anais do XXVI Simpósio Brasileiro de Informática na Educação (SBIE 2015) Maceió-AL, Brasil.

Dantas, A.D.; Guimarães, V.A.; Lima, D.W.F.; Ardaia, M. C. S.; Barreto, W.S.; Castro, T.H.C. - Interaction Evaluation using the Mac-g in Sloodle - 6th International Conference on Software Development and Technologies for Enhancing Accessibility and Fighting Infoexclusion (DSAI 2015) - 160-170 - Elsevier - Procedia Computer -. London - England, 2015.

Dantas, A.D.; Lima, D.W.F.; Maciel, L.C.; De Castro, T.H.C.; Neto, A.R.C.; Lima, I.C. - Avaliação de Comunicabilidade no Soodle - SBSC 2014 - Simpósio Brasileiro de Sistemas Colaborativos - Outubro, 2014.

Fernandes, F., Rodrigues, C. e Werner, C. (2016). Avaliação Heurística de um Ambiente Virtual para Análise de Rotas de Execução de Software. In: IV Workshop on Software Visualization, Evolution and Maintenance - 2016 - Maringá-PR, Brasil.

Fuks, H., Raposo, A.B. and Gerosa, M.A. (2012) "Teorias e modelos de colaboração", In: Sistemas Colaborativos, Organized by Mariano Pimentel and Hugo Fuks, Elsevier, Rio de Janeiro, p. 16-33. 
VI Congresso Brasileiro de Informática na Educação (CBIE 2017)

Anais do XXVIII Simpósio Brasileiro de Informática na Educação (SBIE 2017)

Girotto, V. (2016). Collective Creativity through a Micro-Tasks Crowdsourcing Approach.: In: Computer Supported Cooperative Work - CSCW '16, San FranciscoCA, USA.

Kitchenham, B. (2004) "Procedures for Permorming Systematic Reviews", Joint Technical Report Software Engineering Group, Departament of Computer Science keele University, United king and Empirical Software Engineering, Nacional ICT Australia Ltd, Australia.

Kitchenham, B., Charters, S., Budgen, D., Brereton, P., Turner, M., Linkman,S., Jørgensen, M., Mendes, E. and Visaggio, G. (2007). Guidelines for performing Systematic Literature Reviews in Software Engineering. EBSE Technical Report, Keele University and University of Durham.

Kuntz, V.H., Alves, M. M., Gonzalez, R.D., Ulbricht, V.R., de Macedo, C. M. S. (2013). Contextualização do uso das ferramentas colaborativas em Ambientes Virtuais de Aprendizagem. In: $6^{\circ}$ Congresso Nacional de Ambientes Hipermídia para Aprendizagem - (CONAHPA 2013). João Pessoa-PB, Brasil.

Lima, D. F. M. L., Moraes, C. L. G., Araújo, E.F.M. (2014). Coordenação de Tutoria: Acompanhamento de tutores nos cursos técnicos a distância da UEMA. In: Congresso Internacional ABED de Educação à Distância. Curitiba - PR, Brasil.

Moreira, C., Pimentel, A.R., Maschio, E. (2015). Estudo sobre o Sequenciamento Inteligente e Adaptativo de Enunciados em Programação de Computadores. In: Anais dos Workshops do IV Congresso Brasileiro de Informática na Educação (CBIE 2015). Maceió-AL, Brasil.

Nunes, EP d. S., Roque, LG e Nunes, F. d. L. d. S. (2016). Conhecimento de medição Aquisição em Ambientes de Aprendizagem Virtual 3D. Em Gráficos IEEE Computer e Applications, vol. 36, 2, p. 58-67.

Nunes, F.B., Herpich, F., Paschoal, L.N., Tarouco, L.M.R., de Lima, J.V. Systematic Review of Virtual Worlds applied in Education. In: Anais do XXVII Simpósio Brasileiro de Informática na Educação (SBIE 2016). V Congresso Brasileiro de Informática na Educação (CBIE 2016).

Peixoto, D. C. C., Campos, S. T., Resende, R. F. (2015). Avaliação de Jogos Educacionais Multiusuários: Uma Revisão Sistemática da Literatura. In: Anais do XXVI Simpósio Brasileiro de Informática na Educação (SBIE 2015). Maceió-AL, Brasil.

Santos,N.S., Ferreira, L.S., Prates, R.O.. An Overview of Evaluation Methods for Collaborative Systems - Um Panorama sobre Métodos de Avaliação de Sistemas Colaborativos. In: Brazilian Symposium on Collaborative Systems (SBSC 2012).

Schauer, B. and Zeiller, M. (2011). "E-Collaboration Systems: How Collaborative They Really Are", In: COLLA $1^{\circ}$ The First International Conference on Advanced Collaborative Networks, Systems and Applications, Luxemburgo, p. 16-21. 